\title{
Understanding conserved amino acids in proteins
}

\author{
Nikolay V. Dokholyan, Leonid A. Mirny and Eugene I. Shakhnovich \\ Department of Chemistry, Harvard University, 12 Oxford Street, Cambridge, MA 02138, USA
}

(October 24, 2018)

It has been conjectured that evolution exerted pressure to preserve amino acids bearing thermodynamic, kinetic, and functional roles. In this letter we show that the physical requirement to maintain protein stability gives rise to a sequence conservatism pattern that is in remarkable agreement with that found in natural proteins. Based on the physical properties of amino acids, we propose a model of evolution that explains conserved amino acids across protein families sharing the same fold.

Molecular evolution is a sumptuous natural laboratory that provides an invaluable source of information about the structure, dynamics and function(s) of biomolecules. This information has already been widely used to understand the folding kinetics, thermodynamics, and function of proteins (e.g. [1.,2]). The basic belief behind the majority of such studies is that evolution optimizes, to a certain extent, the properties of proteins, so that they become more sufficiently stable, and have better folding and functional properties.

Recent studies [2, 3] identified positions in several common protein folds where amino acids are universally conserved within each family of proteins having that fold. Such positions are localized in structure, and their unusually strong conservatism may be due to functional reason (e.g. super-site), or folding kinetics (folding nucleus) [1 [7]. In contrast to function and folding kinetics, evolutionary pressure to maintain stability may be applied "more evenly" because all amino acids contribute, to a lesser or greater extent, to protein stability via their interaction with other amino acid residues and with water.

In this letter we develop a model that provides a rationale for conservatism patterns caused by selection for stability. Our model is of equilibrium evolution that maintains stability and other properties achieved at an earlier, prebiotic stage. To this end we propose that stability selection accepts only those mutations that keep energy of the native protein, $E$, below a certain threshold $E_{0}$ necessary to maintain an energy gap 8 11]. The requirement to maintain an energy threshold for the viable sequences makes the equilibrium ensemble of sequences analogous to a microcanonical ensemble. In analogy with statistical mechanics, a more convenient and realistic description of the sequence ensemble is a canonical ensemble, whereby strict requirements on energy of the native state is replaced by a "soft" evolutionary pressure that allows energy fluctuations from sequence to sequence but makes sequences with high energy in the native state unlikely. In the canonical ensemble of sequences, the probability of finding a particular sequence, $\{\sigma\}$, in the ensemble follows the Boltzmann distribution [9 12$]$

$$
P(\{\sigma\})=\frac{\exp (-E\{\sigma\} / T)}{Z},
$$

where $T$ is the effective temperature of the canonical ensemble of sequences that serves as a measure of evolutionary pressure and $Z=\sum_{\{\sigma\}} \exp (-E\{\sigma\} / T)$ is the partition function taken in sequence space.

Next, we apply a mean-field approximation that replaces all multiparticle interactions between amino acids by interaction of each amino acid with an effective field $\Phi$ acting on this amino acid from the rest of the protein. This approximation presents $P(\{\sigma\})$ in a multiplicative form as $\prod_{k=1}^{N} p\left(\sigma_{k}\right)$ of probabilities to find an amino acid $\sigma$ at position $k . p\left(\sigma_{k}\right)$ also obeys Boltzmann statistics

$$
p\left(\sigma_{k}\right)=\frac{\exp \left(-\Phi\left(\sigma_{k}\right) / T\right)}{\sum_{\sigma} \exp \left(-\Phi\left(\sigma_{k}\right) / T\right)} .
$$

The mean field potential $\Phi\left(\sigma_{k}\right)$ is the effective potential energy between amino acid $\sigma$ and all amino acids interacting with it, i. e.

$$
\Phi\left(\sigma_{k}\right)=\sum_{i \neq k}^{N} U\left(\sigma_{k}, \sigma_{i}\right) \Delta_{k i} .
$$

Matrix $U$ describes energy parameters in contact approximation and matrix $\Delta$ is a contact matrix for protein native structure (see Methods for more detail). The potential $\Phi$ is similar in spirit to the protein profile introduced by Bowie et al. 13 to identify protein sequences that fold into a specific $3 \mathrm{D}$ structure.

For each member, $m$, of the fold family (FSSP database 14) we compute the mean-field probability, $p_{m}\left(\sigma_{k}\right)$, using Eq. (2) and then, we compute the average mean-field probability,

$$
p_{M F}\left(\sigma_{k}\right)=\frac{1}{N_{s}} \sum_{m=1}^{N_{s}} p_{m}\left(\sigma_{k}\right) .
$$

Eqs. (2) - (14), along with properly selected energy function, $U$, make it possible to predict probabilities of all amino acid types and sequence entropy $S_{M F}(k)$ at each position $k$

$$
S_{M F}(k)=-\sum_{\sigma} p_{M F}\left(\sigma_{k}\right) \ln p_{M F}\left(\sigma_{k}\right)
$$


from the native structure of a protein. The summation is taken over all possible values of $\sigma$.

Theoretical predictions from statistical-mechanical analysis can be compared with data on real proteins. In order to determine conservatism in real proteins we note that the space of sequences that fold into the same protein structure presents a two-tier system, where homologous sequences are grouped into families and there is no recognizable sequence homology between families despite the fact that they fold into closely related structures [2, 15, 16].

Using the database of protein families with close sequence similarity (HSSP database 17]), we compute frequencies of amino acids at each position, $k$, of aligned sequences, $P_{m}\left(\sigma_{k}\right)$, for a given, $m$ th, family of proteins. We average these frequencies across all $N_{s}$ families sharing the same fold that are present in FSSP database [14]:

$$
P_{a c r}\left(\sigma_{k}\right)=\frac{1}{N_{s}} \sum_{m=1}^{N_{s}} P_{m}\left(\sigma_{k}\right) .
$$

Next, we determine the sequence entropy, $S_{a c r}(k)$, at each position, $k$, of structurally aligned protein analogs:

$$
S_{a c r}(k)=-\sum_{\sigma} P_{a c r}\left(\sigma_{k}\right) \ln P_{a c r}\left(\sigma_{k}\right) .
$$

If the stability selection were a factor in evolution of proteins and our model captures it, then we should observe correlation between predicted mean field based sequence entropies, $S_{M F}(k)$, and actual sequence entropies $S_{a c r}(k)$ in real proteins. Thus, the question is: "Can we find such $T$, so that predicted conservatism profile $S_{M F}(k)$ matches the real one $S_{a c r}(k)$ ?"

By varying the values of the temperature, $T$ in the range $0.1 \leq T \leq 4.0$, we minimize the distance, $D^{2} \equiv$ $\sum_{k=1}^{N}\left(S_{M F}(k)-S_{a c r}(k)\right)^{2}$, between the predicted and observed conservatism profiles. We exclude from this sum such positions in structurally aligned sequences that have more than $50 \%$ gaps in the structural (FSSP) alignment. We denote by $T_{\text {sel }}$ the temperature that minimizes $D$.

We study three folds: Immunoglobulin fold (Ig), Oligonucleotide-binding fold (OB), and Rossman fold (R). We compute correlation coefficient [18] between values of $S_{M F}(k)$, obtained at $T_{\text {sel }}$, and $S_{a c r}(k)$ for all three folds. The results are summarized in Table 1 . The plots of $S_{M F}(k)$ and $S_{a c r}(k)$ versus $k$ as well as their scatter plots are shown in Figs. 1 $3(\mathrm{a}, \mathrm{b})$.

The correlation between $S_{M F}(k)$ and $S_{a c r}(k)$ is remarkable for all three folds and indicates that our mean field model is able to select the conserved amino acids in protein fold families. It is fully expected that the correlation coefficient is smaller than 1 . The reason for this is that computation of $S_{M F}(k)$ takes into account evolutionary selection for stability only and it does not take into account possible additional pressure to optimize kinetic or functional properties.
The additional evolutionary pressure due to kinetic or functional importance of amino acids results in pronounced deviations of $S_{M F}$ from $S_{a c r}$ for few amino acids that may be kinetically or functionally important. A number of amino acids whose conservatism is much greater than predicted by our model form a group of "outliers", from otherwise very close correspondence between $S_{M F}$ and $S_{a c r}$. To demonstrate that some of those amino acids are important for folding kinetics and as such they can be under additional evolutionary pressure, we color data points on $S_{M F}$ versus $S_{a c r}$ scatter plot according to the range of $\phi$-values [19] that corresponding amino acids fall into. Thermodynamics and kinetic role of individual amino acids was studied extensively (i) by Hamill et al. 20 for the TNfn3 (1TEN) protein, and (ii) by López-Hernández and Serrano 21] for the CheY protein. We use the $\phi$-values for individual amino acids obtained in [20,21]. We observe that (i) for TNfn3 protein most of the points on Fig. 1 (b) that belong to the outlier group have $\phi$-values ranging from 0.2 to 1 , and (ii) for CheY protein most of the points (for which $\phi$ values are known) on Fig. 3(b) that belong to the outlier have $\phi$-values ranging from 0.3 to 1 .

To conclude, we presented a theory that explains sequence conservation caused by the most basic and universal evolutionary pressure in proteins to maintain stability. The theory predicts very well sequence entropy for the majority of amino acids, but not all of them. The amino acids that exhibit considerably higher conservatism than predicted from stability pressure alone are likely to be important for function and/or folding. Comparison of the "base-level" stability conservatism $S_{M F}(k)$ with $S_{a c r}(k)$ - actual conservatism profile of a protein fold - allows to identify functionally and kinetically important amino acid residues and potentially gain specific insights into folding and function of a protein.

\section{Methods}

\section{Protein model}

We represent interactions in a protein in a $C_{\beta}$ approximation - each pair of amino acids interact if their $C_{\beta} \mathrm{s}$ $\left(C_{\alpha}\right.$ in the case of Gly) are within the contact distance $7.5 \AA$ [22]. The total potential energy of the protein can be written as follows:

$$
E=\frac{1}{2} \sum_{i \neq j}^{N} U\left(\sigma_{i}, \sigma_{j}\right) \Delta_{i j}
$$

where $N$ is the length of the protein, and $\sigma_{i}$ is the amino acid type at the position $i=1, \ldots, N . U\left(\sigma_{i}, \sigma_{j}\right)$ is the corresponding element of the matrix of pairwise interactions between amino acids $\sigma_{i}$ and $\sigma_{j} . \Delta_{i j}$ is the element 
of the contact matrix that is defined to be 1 if there is a contact between amino acids $i$ and $j$, and is 0 otherwise.

\section{Six-letter code potential}

Due to the similarities in the properties of the 20 types of amino acids one can classify these amino acids into 6 distinct groups: aliphatic $\{A V L I M C\}$, aromatic $\{F W Y H\}$, polar $\{S T N Q\}$, positive $\{K R\}$, negative $\{D E\}$, and special (reflecting their special conformational properties) $\{G P\}$. We construct the effective potential of interaction, $U_{6}\left(\hat{\sigma}_{i}, \hat{\sigma}_{j}\right)$, between six groups of amino acids, $\hat{\sigma}$, by computing the average interaction between these groups, i. e.

$$
U_{6}\left(\hat{\sigma}_{i}, \hat{\sigma}_{j}\right)=\frac{1}{N_{\hat{\sigma}_{i}} N_{\hat{\sigma}_{j}}} \sum_{\sigma_{k} \in \hat{\sigma}_{i}, \sigma_{l} \in \hat{\sigma}_{j}} U_{20}\left(\sigma_{k}, \sigma_{l}\right),
$$

where $\sigma$ denotes amino acids in the 20-letter representation, and $U_{20}\left(\sigma_{k}, \sigma_{l}\right)$ is the 20-letter Miyazawa-Jernigan matrix of interaction [23]; $\hat{\sigma}$ denotes amino acids in the 6 -letter representation. $N_{\hat{\sigma}}$ is the number of actual amino acids of type $\hat{\sigma}$, e. g. for the aliphatic group, $N_{\hat{\sigma}}=6$.

We thank R. S. Dokholyan for careful reading of the manuscript and H. Angerman, S. V. Buldyrev, E. Kussell, L. Li, J. Shimada for helpful discussions. NVD is supported by NIH postdoctoral fellowship. EIS is supported by NIH.

[1] Branden, C. \& Tooze, J., Introduction to protein structure, Garland Publishing Inc., New York, 1998

[2] Mirny, L. A. \& Shakhnovich, E. I., Universally conserved positions in protein folds: reading evolutionary signals about stability, folding kinetics and function. J. Mol. Biol. 291, 177-196 (1999)

[3] Ptitsyn, O. B., Protein folding and protein evolution: Common folding nucleus in different subfamilies of c-type cytochromes? J. Mol. Biol. 278, 655-666 (1998)

[4] Mirny, L. A., Abkevich, V. I. \& Shakhnovich, E. I., How evolution makes proteins fold quickly. Proc. Natl. Acad. Sci. U. S. A. 95, 4976-4981 (1998)

[5] van Nuland, N. A. J., et al., Slow folding of muscle acylphosphatase in the absence of intermediates. J. Mol. Biol. 283, 883-891 (1998)

[6] van Nuland, N. A. J., et al., Slow cooperative folding of a small globular protein hpr. Biochemistry 37, 622-637 (1998)

[7] Dokholyan, N. V., Buldyrev, S. V., Stanley, H. E. \& Shakhnovich, E. I., Identifying the protein folding nucleus using molecular dynamics. J. Mol. Biol. 296, 11831188 (2000)

[8] Sali, A., Shakhnovich, E. I. \& Karplus, M., Kinetics of protein folding. A lattice model study for the requirements for folding to the native state. J. Mol. Biol. 235, 1614-1636 (1994)
[9] Shakhnovich, E. I. \& Gutin, A. M., Engineering of stable and fast folding sequences of model proteins. Proc. Natl. Acad. Sci. U. S. A. 90, 7195-7199 (1993)

[10] Ramanathan, S. \& Shakhnovich, E. I., Statistical mechanics of proteins with evolutionary "selected" sequences. Phys. Rev. E 50, 1303-1312 (1994)

[11] Finkelstein, A. V., Gutin, A. \& Badretdinov, A., Why are the same protein folds used to perform different functions? Proteins 23, 142-149 (1995)

[12] Pande, V. S., Grosberg, A. Yu. \& Tanaka, T., Freezing Transition of random heteropolymers consisting of arbitrary sets of monomers. Phys. Rev. E 51, 3381-3393 (1995)

[13] Bowie, J. U., Luthy, R. \& Eisenberg, D., A method to identify protein sequences that fold into a known 3dimensional structure. Science 253, 164-170 (1991)

[14] Holm, L. \& Sander, C., Protein structure comparison by alignment of distance matrices. J. Mol. Biol. 233, 123138 (1993)

[15] Rost, B., Protein structures sustain evolutionary drift. Folding \& Design 2, S19-S24 (1997)

[16] Tiana, G., Broglia, R. \& Shakhnovich, E. I., Hiking in the energy landscape in sequence space: A bumpy road to good folders. Proteins: Struc. Func. Genet. 39, 244-251 (2000)

[17] Dodge, C., Schneider, R. \& Sander, C., The hssp database of protein structure-sequence alignments and family profiles. Nucl. Acid Res. 26, 313-315 (1998)

[18] Press, W. H., Flannery, B. P., Teukolsky, S. A. \& Vetterling, W. T., Numerical recipes, Cambridge University Press, Cambridge, 1989

[19] Itzhaki, L. S., Otzen, D. E. \& Fersht, A. R., The structure of the transition-state for folding of chymotrypsin inhibitor- 2 analyzed by protein engineering methods evidence for a nucleation-condensation mechanism for protein-folding. J. Mol. Biol. 254, 260-288 (1995)

[20] Hamill, S. J., Steward, A. \& Clarke, J., The folding of an immunoglobulin-like greek key protein is defined by a common-core nucleus and regions constrained by topology. J. Mol. Biol. 297, 165-178 (2000)

[21] López-Hernández, E. \& Serrano, L., Structure of the transition state for folding of the 129 aa protein ChyY resembles that of a smaller protein, CI-2. Folding $\&$ Design 1, 43-55 (1996)

[22] Jernigan, R. L. \& Bahar, I., Structure-derived potentials and protein simulations. Curr. Opinion Struc. Biol. 6, 195-209 (1996)

[23] Miyazawa, S. \& Jernigan, R. L., Residue-residue potentials with a favorable contact pair term and an unfavorable high packing density term, for simulation and threading. J. Mol. Biol. 256, 623-644 (1996)

[24] Bernstein, F. C., et al., The Protein Data Bank: a computer-based archival file for macromolecular structures. J. Mol. Biol. 112, 535-542 (1977)

[25] Abola, E. E., Bernstein, F. C., Bryant, S. H., Koetzle, T. F. \& Weng, J. Protein Data Bank. in Allen, F. H., Bergerhoff, G. \& Sievers, R., eds., Crystallographic Databases-Information Content, Software Systems, Scientific Applications, pp. 107-132. Data Commission of the International Union of Crystallography, Cambridge 1987. 


\begin{tabular}{|c|c|c|c|c|c|}
\hline Fold & $N_{s}$ & \begin{tabular}{|l} 
Representative p \\
PDB code 24, 25
\end{tabular} & $\begin{array}{c}\text { otein } \\
N\end{array}$ & $\begin{array}{l}\text { Correlation coefficient } \\
S_{M F}(k) \text { vs. } S_{a c r}(k)\end{array}$ & $T_{s e l}$ \\
\hline$\overline{\mathrm{Ig}}$ & 51 & $1 T E N$ & 89 & 0.63 & 0.34 \\
\hline $\mathrm{OB}$ & 18 & $1 M J C$ & 69 & 0.69 & 0.19 \\
\hline $\mathrm{R}$ & 166 & $3 C H Y$ & 128 & 0.71 & 0.25 \\
\hline
\end{tabular}

TABLE I. The values of the correlation coefficient $r$ for the linear regression of $S_{M F}(k)$ versus $S_{a c r}$ for $\mathrm{Ig}, \mathrm{OB}$, and R, folds and the corresponding optimal values of the temperature $T=T_{\text {sel }}$.

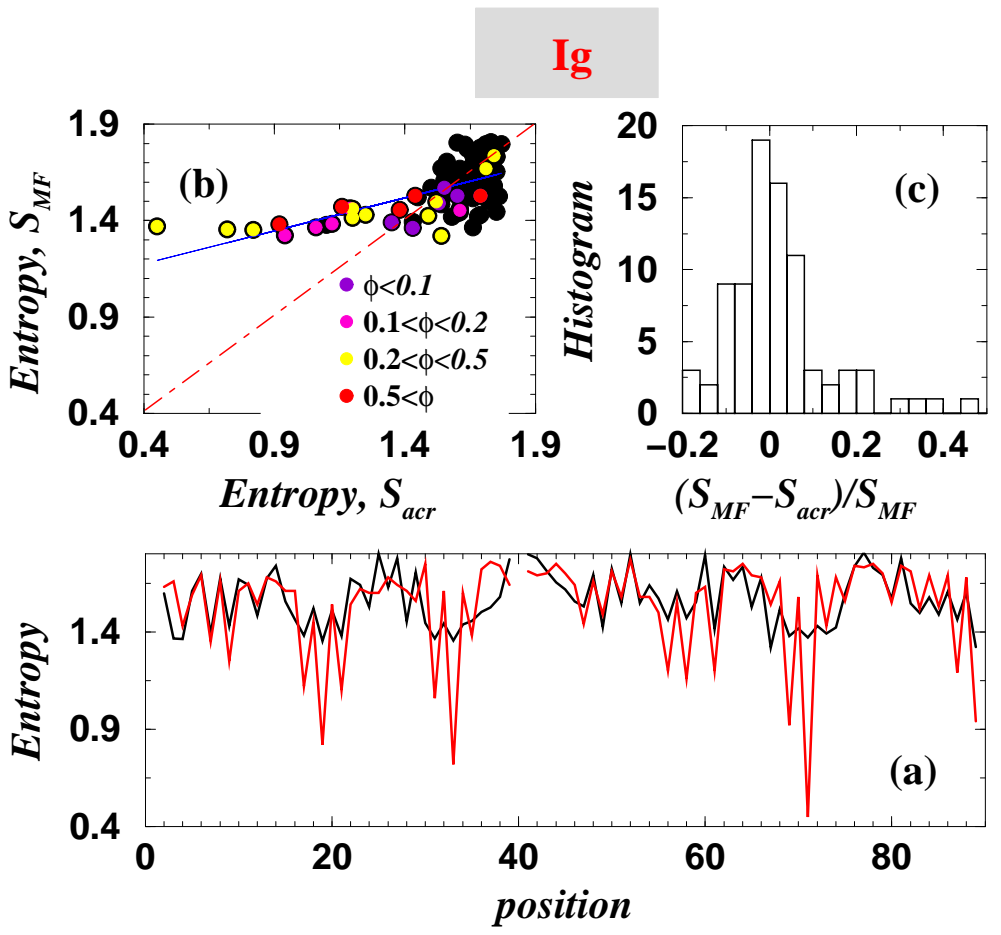

FIG. 1. (a) The values $S_{M F}(k)$ (black line) and $S_{a c r}(k)$ (red line) for all positions, $k$, for the Ig-fold. The lower the values of $S_{M F}(k)$ the more conservative amino acids are at these positions. (b) The scatter plot of predicted $S_{M F}(k)$ versus observed $S_{a c r}(k)$. The linear regression correlation coefficients are shown in Table If. Blue line is the linear regression has the slope different than 1 (red line), corresponding to the $S_{M F}(k)=S_{a c r}(k)$ relation. (c) The histogram of the differences between $S_{M F}(k)$ and $S_{a c r}(k)$. In (b) we assign colors to data points corresponding to amino acids with the specific range of $\phi$-values 20: red, if $0.5<\phi<1$, yellow, if $0.2<\phi<0.5$, magenta, if $0.1<\phi<0.2$, violet if $\phi<0.1$, and black if $\phi$-values are not determined. 

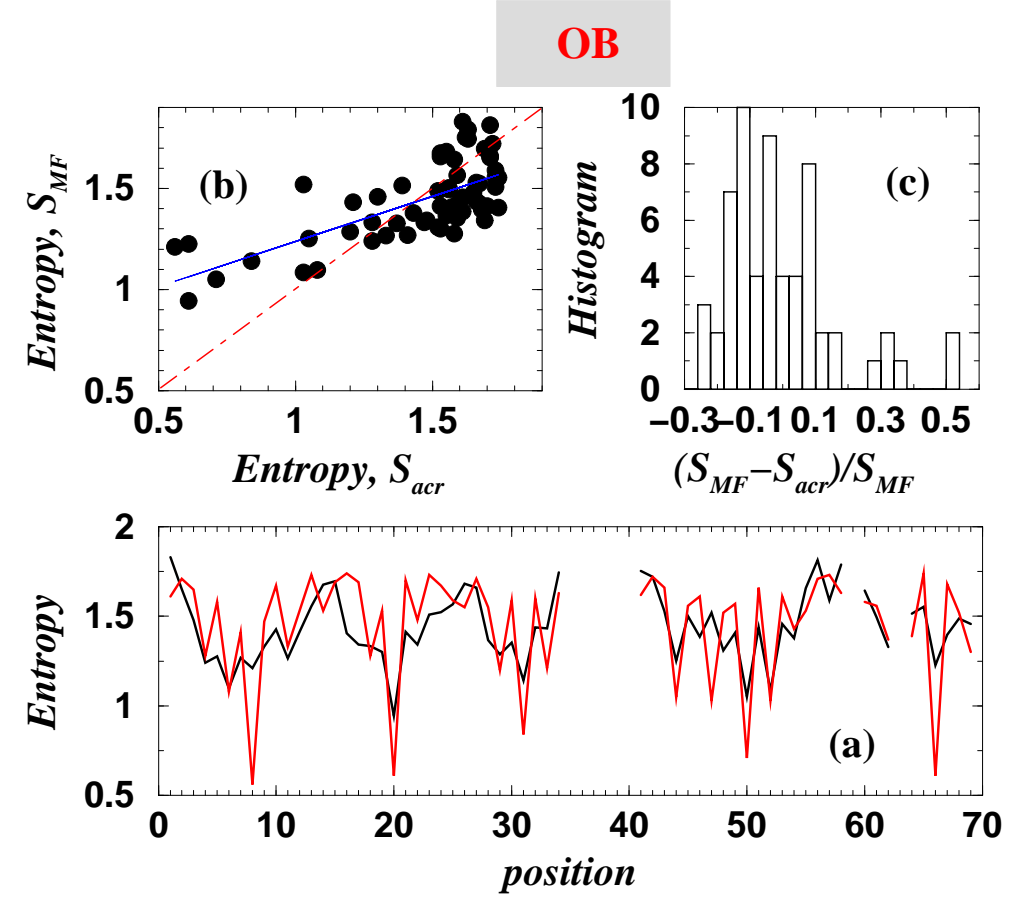

FIG. 2. (a) - (c) The same as Fig.1 1 but for the OB-fold.

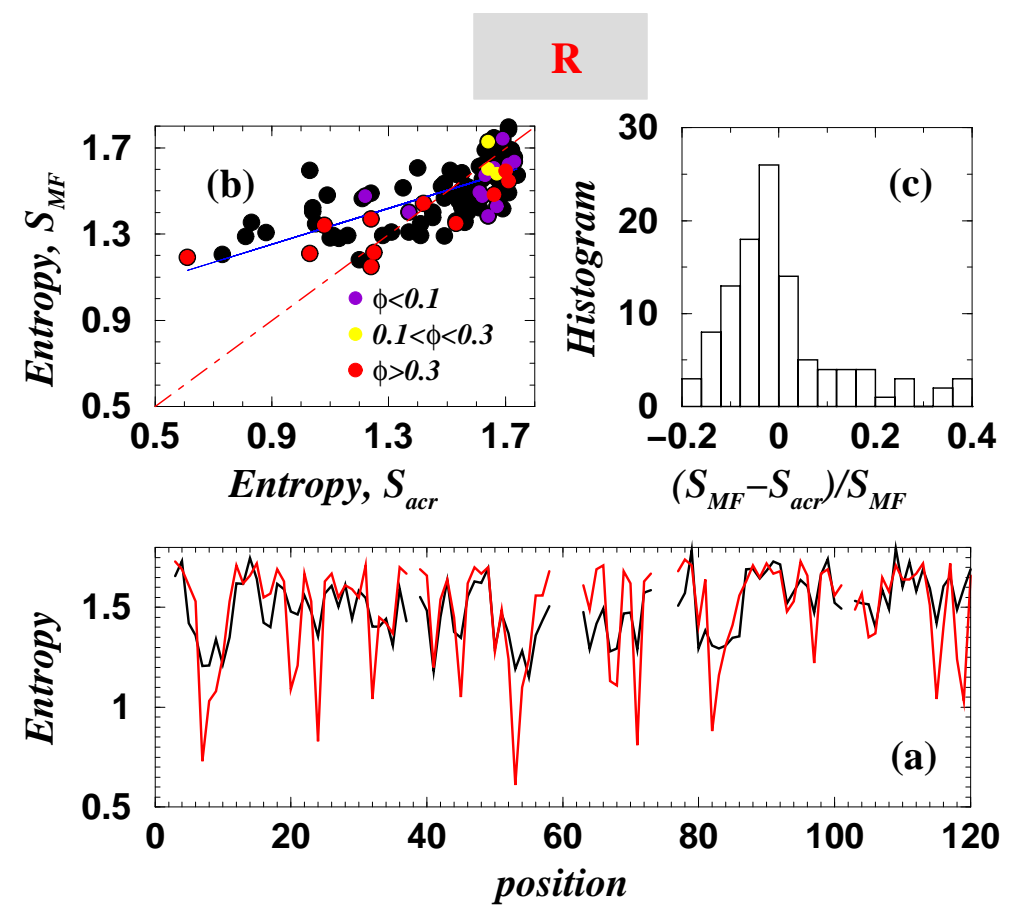

FIG. 3. (a) - (c) The same as Fig.11 but for R-fold. In (b) we assign colors to data points corresponding to amino acids with the specific range of $\phi$-values [21]: red, if $0.3<\phi<1$, yellow, if $0.1<\phi<0.3$, violet if $\phi<0.1$, and black if $\phi$-values are not determined. 